\title{
A Study on The Genesis of " $f$ " Waves of Atrial fibrillation in Man Considered as Multiple Statistical Time Series
}

\author{
Etsu Hashida, M.D., * Naohiro Yoshrtani, Ph.D.,** \\ and Takenobu TAsaki, Ph.D.**
}

\section{Summary}

The fluctuations of base line of the electrocardiogram (ECG) in man with chronic atrial fibrillation ( $f$ waves) were considered as random signals. Simultaneously recorded ECGs from several points on the chest wall, in the esophagus and the right intracardiac cavities were regarded as multiple statistical time series. A spectral matrix was computed from them, and the number and location of the $f$ wave generators and the direction of the circulating excitation wave were estimated or determined by principal component analysis of the spectral matrix, and further multiple and partial coherences between the $f$ waves of simultaneous ECGs were computed. Then, the following conclusions were obtained: 1. The number of generators for the $f$ waves was one and this generator was apparently located somewhere in the right atrium. Stimuli from here generated a circulating excitation wave which descended in the right atrium and ascended in the left. Further, the number of generators was one, which was clearly interpreted from the values of multiple coherences. Therefore, it was evidently demonstrated that $f$ waves were produced by neither ectopic impulse formation nor microre-entry but by macrore-entry (circus movement) in addition to the presence of one generator (focus), as supported also by mere inspection of simultaneously recorded EGGs. 2. In reference to the results of computer simulation conducted hitherto by several authors, it was postulated that atrial fibrillation was initiated by an appropriate premature beat and the perpetuation of this arrhythmia was maintained by a circulating excitation wave, but from the present investigation and the theory recently advanced by Cranefield, one focus persistently sending out impulses at a certain frequency in one direction only could be thought necessary for the initiation and possibly for the perpetuation of atrial fibrillation.

\section{Additional Indexing Words:}

Ectopic focus

Arrhythmia
Electrocardiogram

Circus movement
Electrophysiology of the heart

Computer simulation

From the Department of Internal Medicine, Health Insurance Hospital of Shiga, Ohtsu,* and the Computing Center, Shionogi Research Laboratory, Osaka***

Address for reprint: Etsu Hashida, M.D., Health Insurance Hospital of Shiga, Fujimidai, Ohtsu, Japan 520.

Received for publication March 20, 1979.

Manuscript revised August 2, 1979. 


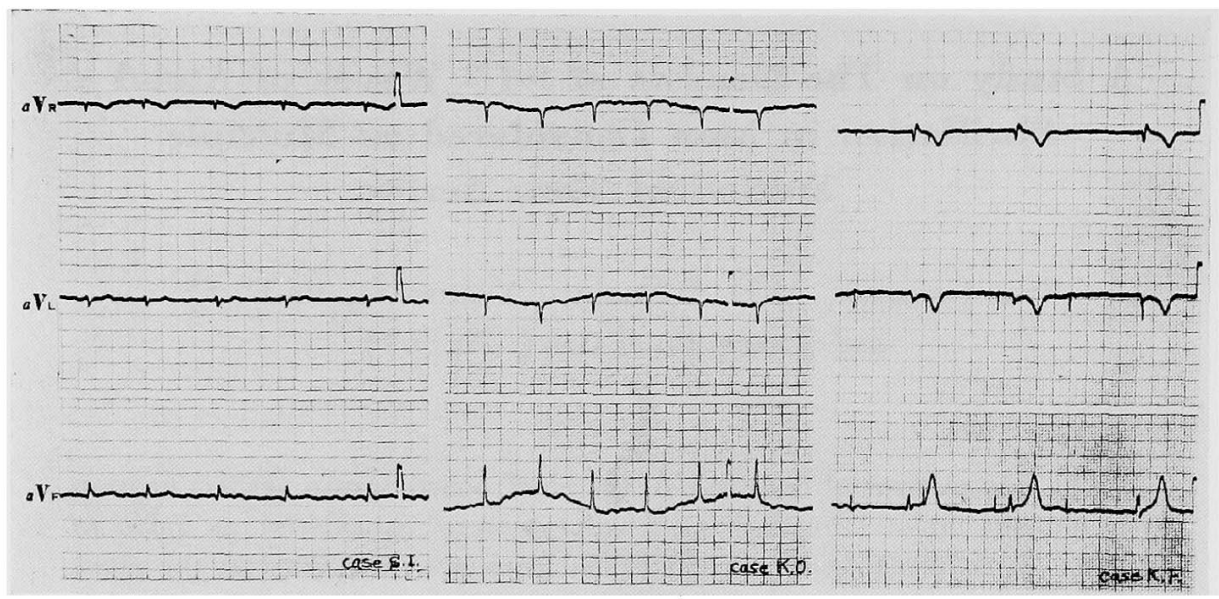

Fig. 1. Simultaneous 3-lead electrocardiogram of leads aVR, aVL and aVF. A peak or a trough of the $f$ waves in leads aVR and aVL is synchronous with a trough or a peak of the $f$ waves in lead aVF. In a third case (case K.F.) pacemaking artefacts are noted.

$\mathrm{A}^{\mathrm{S} \text { the cause of atrial fibrillation in man, the re-entry hypothesis }{ }^{1 / 2)} \text { seems }}$ ported. Also the mechanism of its initiation is not so clear. When we observe simultaneously recorded electrocardiograms (ECGs) of atrial fibrillation minutely, we can consistently find that a peak or a trough of the $f$ waves of leads aVR and aVL corresponds to a trough or a peak of those of lead aVF, as is shown in Fig. 1. This corresponds to the presence of phase difference of about $180^{\circ}$ between the lead locations of aVR and aVL, and that of $\mathrm{aVF}$ from the standpoint of the macrore-entry hypothesis. This fact may be explained neither by microre-entry hypothesis which emphasizes that the excitation wave front propagates quite irregularly, nor by ectopic focus hypothesis which considers that impulses from one focus spread out radially in all directions.

It is the aim of the present investigation to estimate or determine the mechanism of production of the $f$ waves, the direction of the circulating excitation wave and the number and location of the $f$ wave generators by regarding the fluctuations of base line of the ECG in atrial fibrillation ( $f$ waves) as random signals and treating simultaneous 4 lead ECGs recorded from several points as multiple statistical time series. ${ }^{8}$ ) Because loss of information when using only the chest leads should be reflected upon, both esophageal and intracardiac leads were recorded together with chest leads in a third case. 

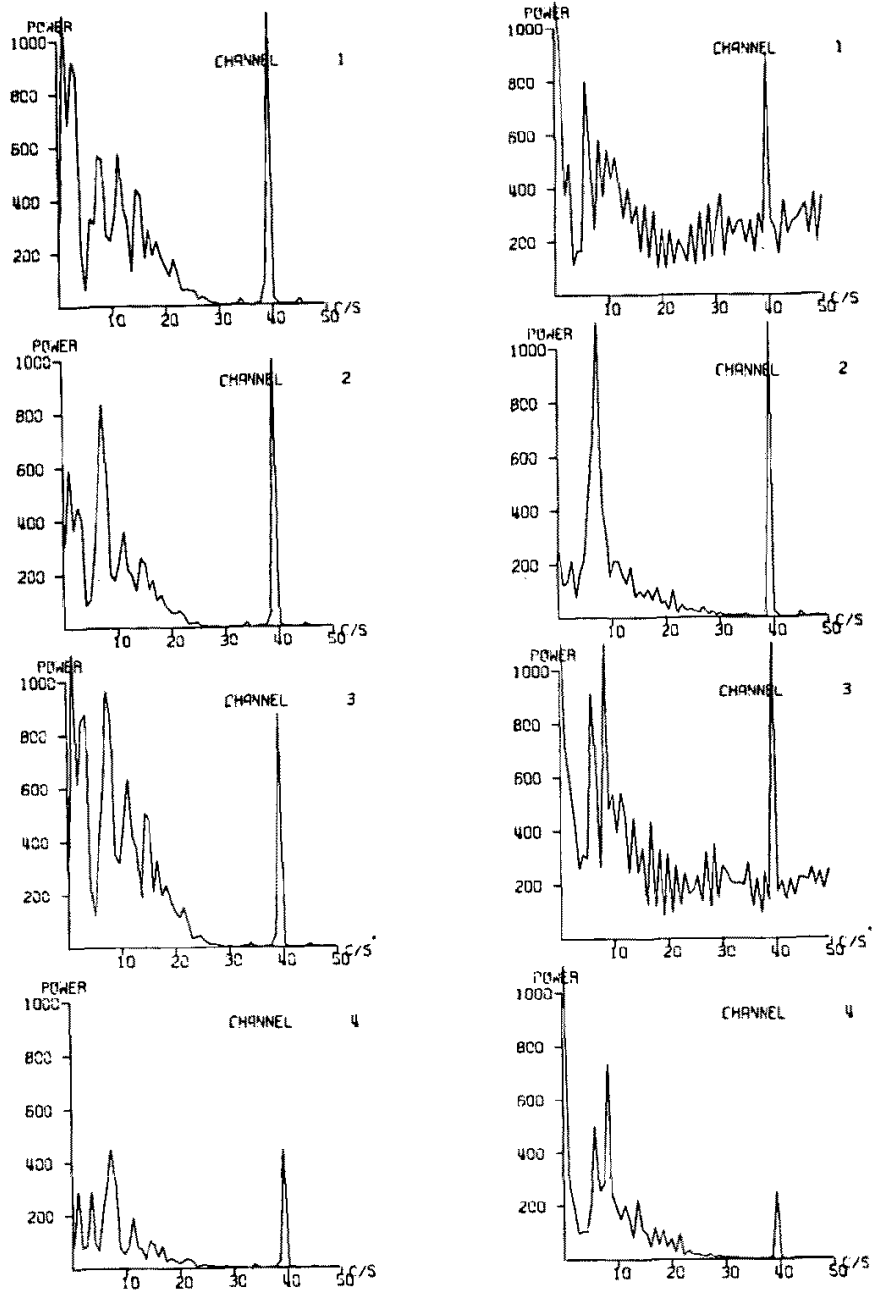

Fig. 2. The left figure illustrates the spectra of the $f$ waves of Case S.I. and the right one, those of Case K.O. The spectra (in arbitrary units) are plotted against frequency (cps). In each case, channels 1, 2, 3 and 4 represent the spectra when the different electrode is placed at the location corresponding to lead $V_{1}$, lead $V_{3 R}$, the midway between the location of lead $V_{1}$ and the xiphoid, and the xiphoid, respectively. There is a large peak at $8 \mathrm{cps}$ common to spectra from each lead location.

\section{Materials and Methods}

The objects were simultaneous ECGs of a 55-year-old male patient (S.I.), a 82-year-old male patient (K.O.), and a 74-year-old male patient (K.F.) with chronic atrial fibrillation. The simultaneous 3-lead (aVR, aVL, and aVF) ECGs of these patients are shown in Fig. 1. The right hand was used as a remote electrode and 
Table I. Summary of Generator

\begin{tabular}{l|c|c|c|c}
\hline & & $\begin{array}{r}\text { Location of the } \\
\text { electrodes } \\
\text { Contribution } \\
\text { ratio of the } \\
\text { 1st principal } \\
\text { component (\%) }\end{array}$ & V & Xiphoid \\
\hline \multirow{3}{*}{ Case S.I. } & Array 1 & 94.78 & 3.82 & 8.78 \\
& Array 2 & 96.14 & & 9.46 \\
Case K.O. & Array 3 & 96.14 & & 24.77 \\
& Array 1 & 92.53 & 4.00 & 13.18 \\
& Array 2 & 89.97 & & 42.03 \\
& Array 3 & 83.48 & & 74.55
\end{tabular}

The locations of the electrodes are shown in the first row. The contribution ratio of the first (largest) principal component to the cumulative sum of the eigenvalues is represented in the third

4 points on the chest wall were used to compose different electrodes. Simultaneous recording was made with an electroencephalograph in several arrays of electrodes, and also on magnetic tape at the same time. Analog-digital (A-D) transform of the data was made for about $8 \mathrm{sec}$ in the first 2 cases and about $5 \mathrm{sec}$ in a third case at a sampling interval of $10 \mathrm{msec}$. Inspection of the spectra indicated a large peak common to each simultaneous 4-lead ECG at about $8.0 \mathrm{~Hz}$ in the first 2 cases and at about $6.0 \mathrm{~Hz}$ in a third case, so that a continuous record including both QRS complex and $\mathrm{T}$ wave can be used as the object of analysis.

The mathematical procedures used in what follows are similar to those in the case of atrial flutter.

\section{Results}

The center frequency common to each simultaneous 4-lead ECG was determined as $8.0 \mathrm{~Hz}$ in the first 2 cases from inspection of ECGs recorded at each location and the results from which spectra of the $f$ waves at each recording location were plotted against frequency. The results of generator analysis at the above center frequency are shown in Table I. The spectra in Array 1 is shown, as an example, in Fig. 2. As the contribution ratio of the largest eigenvalue to the cumulative sum of the eigenvalues in each array was over $90 \%$ in almost all arrays as seen in Table $I$, the number of $f$ wave generators can be regarded as one.

Table I shows further the phase lags between the location of generator and each recording site at a center frequency of $8.0 \mathrm{~Hz}$. It is clear in Array 1 that the excitation wave proceeds from the location corresponding to lead $V_{1}$, via the midway between this point and the xiphoid, to the xiphoid. We can see in Array 2 that the excitation wave proceeds from the location cor- 
Analysis of the First 2 Cases

\begin{tabular}{|c|c|c|c|c|c|}
\hline $\begin{array}{l}\text { Midway between } \\
\text { xiphoid and lead } \\
V_{1}\end{array}$ & $V_{1}$ & $V_{2}$ & $V_{3}$ & $\mathrm{~V}_{4}$ & $V_{5}$ \\
\hline-0.20 & -3.22 & -13.97 & $\begin{array}{r}-18.21 \\
0.42\end{array}$ & $\begin{array}{r}-0.50 \\
15.05\end{array}$ & 31.83 \\
\hline 0.05 & -21.96 & -81.11 & $\begin{array}{l}-59.55 \\
-23.61\end{array}$ & $\begin{array}{r}0.73 \\
-1.43\end{array}$ & 54.80 \\
\hline
\end{tabular}

column. The numbers from the fourth column on show in units of degrees phase angles of each array in each row respectively.

responding to lead $V_{2}$ to the xiphoid. Because $f$ waves in leads $V_{4}$ and $V_{5}$ are very small, the state of excitation in the left atrium can not be accurately determined from the present investigation. It is likely, however, that the excitation wave proceeds from the xiphoid to the left atrium, as will be stated later based on the data of a third case. The ranges of phase angles of about $75^{\circ}$ to $180^{\circ}$ and $-75^{\circ}$ to $-180^{\circ}$ seem to correspond to the excitation in the left atrium (Case K.O.). In Case S.I., the ranges of phase angles corresponding to the left atrium are much larger. The fact that the phase angles of the $f$ waves in leads aVR and aVL are almost identical from mere inspection could be explained by the assumption that the $f$ waves in both leads are due to the potential variations of the upper part of the anterior chest wall.

In summarizing the results of the first 2 cases, the number of $f$ wave generators is one. The generator could be assumed to be situated somewhere in the right atrium from the values of the phase angles (as the phase angles over the right atrium are small), and the excitation wave which generates $f$ waves is considered to descend in the right atrium and ascend in the left.

As shown in Table II, the values of multiple coherences indicated values near unity in every array and partial coherences showed rather large values between 2 adjacent locations in at least some arrays.

In a third case, the channels 1, 2, 3, and 4 recorded the ECGs when the different electrode was placed respectively at the right sternal border in the 4 th intercostal space, distally in the esophagus, more orally (about $6 \mathrm{~cm}$ ) than the preceding position and in the right intracardiac cavities.

The position of intracardiac electrode in each array is shown in Table III and illustrated in Fig. 3. Each figure 1-8 in Table III corresponds to 
Table II. Squared Coherences of each Array

\begin{tabular}{l|cccc|cccc}
\hline \multirow{4}{*}{ Array 1 } & 0.979 & 0.631 & 0.876 & 0.826 & 0.996 & 0.226 & 0.921 & 0.707 \\
& 0.222 & 0.973 & 0.918 & 0.868 & 0.823 & 0.945 & 0.452 & 0.520 \\
& 0.763 & 0.597 & 0.995 & 0.927 & 0.971 & 0.762 & 0.998 & 0.878 \\
& 0.613 & 0.249 & 0.675 & 0.988 & 0.672 & 0.353 & 0.740 & 0.977 \\
\hline \multirow{4}{*}{ Array 2 } & 0.964 & 0.920 & 0.908 & 0.832 & 0.649 & 0.469 & 0.492 & 0.623 \\
& 0.592 & 0.971 & 0.921 & 0.867 & 0.054 & 0.936 & 0.856 & 0.743 \\
& 0.104 & 0.086 & 0.972 & 0.942 & 0.068 & 0.753 & 0.950 & 0.793 \\
& 0.180 & 0.229 & 0.352 & 0.957 & 0.087 & 0.418 & 0.517 & 0.912 \\
\hline \multirow{4}{*}{ Array 3 } & 0.994 & 0.975 & 0.913 & 0.931 & 0.888 & 0.739 & 0.445 & 0.565 \\
& 0.811 & 0.991 & 0.858 & 0.944 & 0.695 & 0.967 & 0.495 & 0.845 \\
& 0.666 & 0.395 & 0.965 & 0.871 & 0.046 & 0.226 & 0.595 & 0.384 \\
& 0.093 & 0.184 & 0.028 & 0.962 & 0.555 & 0.859 & 0.172 & 0.942
\end{tabular}

The values of multiple coherences are shown in the diagonal, those of simple coherences, in the right upper part and those of partial coherences, in the left lower part. The data of Ciase S.I. are on the left and those of Case K.O., on the right.

From up to downwards, recording locations for each array are as follow: Array 1; location corresponding to lead $V_{1}\left(V_{1}\right)$, location corresponding to lead $V_{3 R}\left(V_{3 R}\right)$, location midway between xiphoid and $V_{1}$, and xiphoid; Array 2 : location corresponding to lead $V_{2}\left(V_{2}\right)$, location corresponding to lead $V_{3}\left(V_{3}\right)$, location corresponding to lead $V_{4}\left(V_{4}\right)$ and xiphoid; Array $3: V_{3}, V_{4}$, location corresponding to lead $V_{5}\left(V_{5}\right)$ and xiphoid.

Table III. Summary of Generator Analysis of the Third Case

\begin{tabular}{|c|c|c|c|c|c|c|}
\hline & $\begin{array}{l}\text { Position of the } \\
\text { intracardiac } \\
\text { electrode }\end{array}$ & $\begin{array}{l}\text { Contribution } \\
\text { ratio of the } \\
\text { largest } \\
\text { eigenvalue } \\
(\%)\end{array}$ & $\begin{array}{l}\text { Phase angle } \\
\text { of the elec- } \\
\text { trode at the } \\
\text { right } 4 \text { th } \\
\text { interspace }\end{array}$ & $\begin{array}{l}\text { Phase angle } \\
\text { of the elec- } \\
\text { trode at the } \\
\text { distal part } \\
\text { of the } \\
\text { esophagus }\end{array}$ & $\begin{array}{l}\text { Phase angle } \\
\text { of the elec- } \\
\text { trode at the } \\
\text { oral part } \\
\text { of the } \\
\text { esophagus }\end{array}$ & $\begin{array}{l}\text { Phase angle } \\
\text { of the elec- } \\
\text { trode placed } \\
\text { in the } \\
\text { cardiac } \\
\text { cavity }\end{array}$ \\
\hline Array 1 & $\begin{array}{l}\text { in the right ventricle, } \\
\text { lower part }\end{array}$ & 88.33 & -0.18 & 45.77 & 66.12 & -51.39 \\
\hline Array 2 & $\begin{array}{l}\text { in the right atrium, } \\
\text { lower part }\end{array}$ & 90.51 & 0.08 & 18.73 & 34.41 & -8.01 \\
\hline Array 3 & $\begin{array}{l}\text { in the right atrium, } \\
\text { upper lateral part }\end{array}$ & 95.54 & 51.29 & 57.55 & 71.12 & 0.01 \\
\hline Array 4 & $\begin{array}{l}\text { in the superior vena } \\
\text { cava, } \\
\text { lower part }\end{array}$ & 88.41 & -21.76 & 16.55 & 45,11 & 0.18 \\
\hline Array 5 & $\begin{array}{l}\text { in the right atrium, } \\
\text { lower lateral part }\end{array}$ & 86.12 & -0.18 & 10.58 & 20.91 & -39.76 \\
\hline Array 6 & $\begin{array}{l}\text { in the right atrium, } \\
\text { middle lateral part }\end{array}$ & 86.41 & 0.10 & -33.43 & 16.77 & -46.05 \\
\hline Array 7 & $\begin{array}{l}\text { in the right atrium, } \\
\text { upper lateral part }\end{array}$ & 89.20 & 0.19 & -83.23 & 29.46 & 28.19 \\
\hline Array 8 & $\begin{array}{l}\text { in the right atrium, } \\
\text { upper lateral part }\end{array}$ & 82.52 & 46.81 & -29.14 & 5.81 & 0.75 \\
\hline
\end{tabular}

The locations of the intracardiac electrodes are illustrated in the second column and in Fig. 3. The contribution ratio of the first (largest) principal component to the cumulative sum of the eigenvalues is represented in the $3 \mathrm{rd}$ column. The numbers from the 4 th column on indicate in units of degrees phase angles of each array, respectively. 


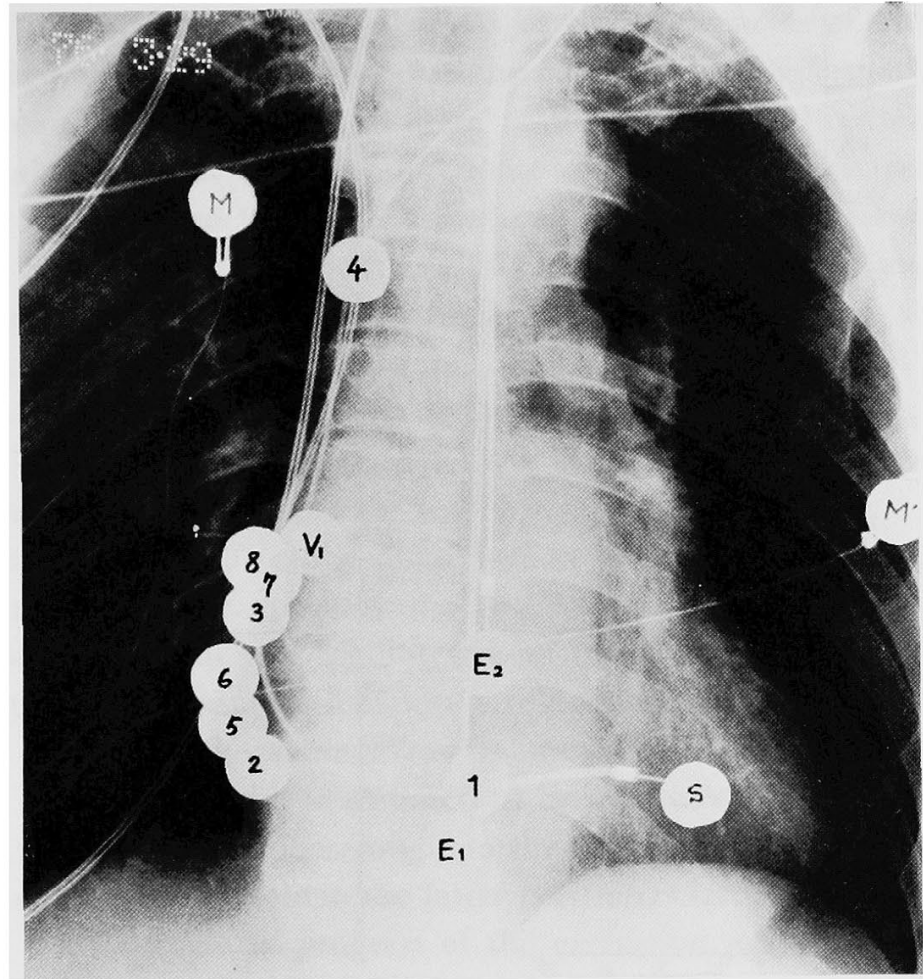

Fig. 3. Roentgenogram of a third case (patient K.F.), a 74-year-old man suffering from atrial fibrillation with a very slow ventricular response who was implanted with an intracardiac pacemaker. The white circles with a figure and letter within indicate the approximate location of the intracardiac, esophageal and chest wall electrode at the time the electrocardiographic record was madc. $M$ and $M^{\prime}$ indicate the electrodes on the chest wall for monitoring purpose. $\mathrm{E}_{1}$ and $\mathrm{E}_{2}$ indicate the esophageal electrodes. $\mathrm{S}$ indicates an electrode implanted in the right ventricle for the pacemaking. $V_{1}$ indicates the electrode located at the right sternal border in the 4 th intercostal space.

the same figure as in Fig. 3. The esophageal electrode in Arrays 1-4 is located at the position intermediate between the 10 th and the 11 th thoracic vertebra in the channel 2 and between the 8 th and the 9th thoracic vertebra in the channel 3, respectively. In Arrays 5-8, the esophageal electrode is located at the position intermediate between the 8 th and the 9 th thoracic vertebra, and between the 6 th and the 7 th thoracic vertebra, respectively. In this case, ECGs were recorded for about $5 \mathrm{sec}$ simultaneously with a magnetic tape recording, and the same procedures of computation as in the first two cases were performed. A sample length of about $5 \mathrm{sec}$ in this case seems to be short for computation of a stationary time series, but a frequency spectrum analyzer of the electroencephalogram dealing with records of frequencies 

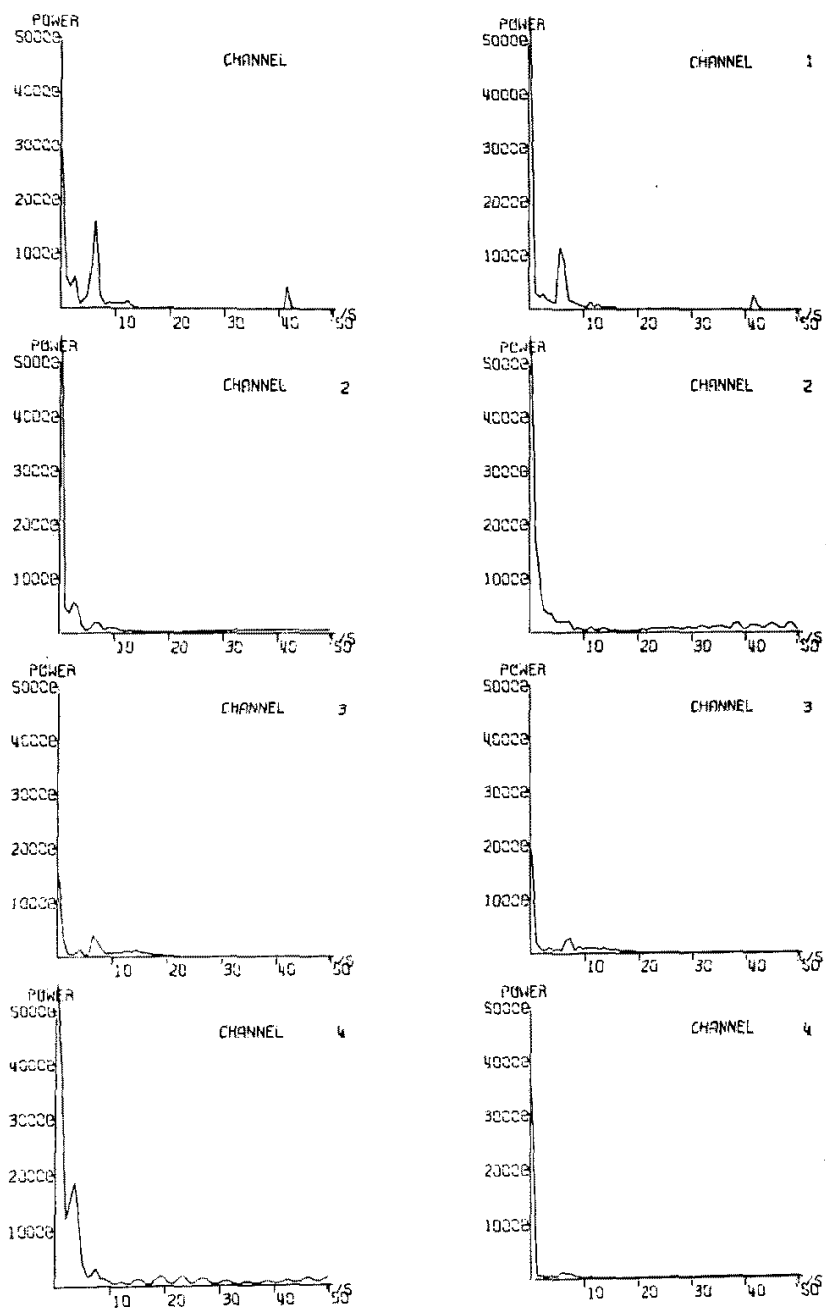

Fig. 4. On the left the spectra of Array 1, and on the right those of Array 2 are shown. Note that the peaks of the spectra of Array 1 are located at the frequency greater than $6.0 \mathrm{~Hz}$ and those of Array 2 are located at the frequency smaller than $6.0 \mathrm{~Hz}$. In Array 1 the electrode placed in the right intracardiac cavity was in the right ventricle, and in Array 2 the electrode was located at the lower part of the right atrium.

similar to $f$ waves is generally set to the period of 5 or $10 \mathrm{sec}$ as the sample length of analysis, and also the sample length of about $6 \mathrm{sec}$ can be calculated according to the theory of Blackman and Tukey.9" Therefore, the sample length of about $5 \mathrm{sec}$ is not too short.

The results of spectral analysis in the third case indicated the presence of peak at about $6.0 \mathrm{~Hz}$, but the peaks of the spectra fluctuate slightly around $6.0 \mathrm{~Hz}$ as the case may be. The spectra of this case are illustrated in Fig. 4. 
Therefore, principal component analysis at the frequencies of both $5.86 \mathrm{~Hz}$ and $6.64 \mathrm{~Hz}$ was performed. Based on the magnitude of the largest eigenvalue at these frequencies and the inspection of the spectra, the center frequency for the analysis was determined and these center frequencies were $5.86 \mathrm{~Hz}$ except for Array 1 and 8. With either one of these frequencies as a center frequency, principal component analysis together with coherence anlaysis was carried out. The results of principal component analysis is shown in Table III. As indicated in this table, the contribution ratio of the largest eigenvalue to the cumulative sum of the eigenvalues is over $80 \%$ in all the arrays. Therefore, the number of $f$ wave generators in this case could be regarded as one.

Furthermore, in this table, phase lags between the location of generator and each recording site are shown. The phase angle of each array at the right sternal border in the 4th intercostal space is generally small. Even when the phase angle at this location is relatively large, that in the right intracardiac cavity in the same array is small. Therefore, the location of generator, if present, could be assumed to be somewhere in the right atrium. Moreover, comparing the phase angle in the distal esophagus with that in the oral position, the phase angle in the latter position is larger than in the former. Therefore, the direction of progress of the excitation wave in the esophagus can be assumed to be caudo-cranial.

As indicated in Table III, the phase differences between the 2 positions in the esophagus in Arrays 5-8 whose esophageal electrodes are located more orally are generally much greater than those in Arrays 1-4. This fact might be due to the following reason: Because the locations of the electrodes in Arrays 5-8 correspond to the upper posterior portion of the left atrium, the excitation wave recorded at these locations might be considered to progress orally (centrifugally) as a daughter wave emanating from a so-called mother circus ring. However, the position of the esophageal electrodes in Array 5 is the same as that in Arrays 6-8. Therefore, the presence of large phase difference in these arrays is difficult to understand and so awaits further investigation.

As for the values of coherences, their behaviors are nearly identical to those of the first 2 cases. Therefore, a table of coherences of this case is omitted.

\section{Discussion}

Our results demonstrate that one generator is located somewhere in the right atrium and a circulating excitation wave proceeds in the right atrium 
in a cranio-caudal direction. The course of propagation in the left atrium is not clear, for our results were based on leads from limited areas. Lack of leads from the left atrium may be the weak point in the present study as Rytand $^{10)}$ stressed, and this situation is common to the report of atrial flutter by Lewis et al. ${ }^{11)}$ However, because of the reasons previously mentioned on atrial flutter, our study employed only leads from the body surface in the first 2 cases. Application of the present method of analysis to the ECGs including esophageal and/or intracardiac EGGs was performed in a third case. Therefore the present report is only a preliminary one. In this preliminary investigation the progress of excitation wave front was demonstrated to be caudocranial in the esophagus. Further, Lewis ${ }^{12}$ observed the rotation of the electrical axis in clinical cases, and stated that he could explain $f$ waves appropriately by assuming a single circuit which showed a rotation of $360^{\circ}$ in each period of the $f$ waves through an atrial path with some width. Lewis et al $^{13)}$ regarded the left atrium as the supposed path of the circulating wave in atrial flutter. If similar assumption is possible in atrial fibrillation, the ranges of phase angle of about $-180^{\circ}$ to $-75^{\circ}$ and $75^{\circ}$ to $180^{\circ}$ (Case K.O.) can be regarded as corresponding to the left atrium. The change of the phase angles was below $30^{\circ}$ over the right atrium and the excitation wave could be regarded as proceeding in a cranio-caudal direction as seen from their signs $\left(+\right.$ or - ). Therefore, if a rotation of $360^{\circ}$ is postulated, the path should be one which descends in the right atrium and ascends in the left. Such a direction of rotation is the same with the case of common type of atrial flutter.

Further, atrial fibrillation is known to be not maintained in animal experiments when function of the sinus node was suppressed as Sano et al ${ }^{14}$ and Nadeau et al ${ }^{15}$ indicated. And also Scherf and Cohen ${ }^{5}$ stated that "these attacks of fibrillation were promptly stopped by simultaneous cooling of the sinus and of the AV node areas". Thus, both the sinus node and the AV node or the sinus node alone are probably involved in the initiation and perpetuation of this arrhythmia irrespective of whether either circus movement hypothesis or ectopic center hypothesis is valid. Further, it is also reasonable to assume the presence of a definite path of circulating excitation wave as in the case of WPW syndrome from a study of familial occurrence of atrial fibrillation. ${ }^{16)}$

The mechanism of initiation of atrial fibrillation can be considered similar to that of atrial flutter. The centrencephalon hypothesis ${ }^{17}$ ) will also serve as a reference.

The necessity of premature beat in the mechanism of initiation of atrial fibrillation should be postulated also from computer simulation. 
Wiener and Rosenblueth ${ }^{18)}$ proposed a theory to solve the problems on the conduction of impulses in neural network and in syncytium of cardiac muscle. Computer simulation was conducted according to this theory by Farley and Clark ${ }^{19}$ ' and Farley ${ }^{201,21)}$ on the brain wave, and by Moe et al ${ }^{22)}$ on atrial fibrillation. The results of these studies were essentially similar in dealing with the fluctuations of the base line. These studies postulated appropriate stimulation for the initiation, and reverberation (for brain wave) or re-entry (for ECG) for the maintenance. In a model by Moe et al, introduction of an obstacle into the network results in a periodic circus movement flutter. This suggests a close relationship between atrial fibrillation and flutter, and postulates the presence of a circulating excitation wave in atrial fibrillation in a wider sense.

Similar results were obtained by a more intuitional calculation by Griffith. ${ }^{23)}$ The outline of his theory is as follows: In the case in which several identical neurons having only excitatory combination are linked together at random and in which there is no input but an arbitrarily assigned initial activity, he made simple calculation for the study of the behavior of the neural network. As a result, he indicated that any initial activity tended to disappear while an initial activity was less than a certain threshold level and it tended to the fully active state while an initial activity was greater than it. Thus, the neural network has a threshold value for its mean activity, so that it is shown that the activity is self-maintained and the whole network continues to be excited if an initial activity is greater than this threshold, and that the activity rapidly becomes quiescent if the value of an initial activity is less than this threshold.

However, construction of a network which does not have such a property is also possible. For instance, such a network can be obtained by constructing a neural network which consists of 2 totally disconnected parts; one part could persist in the inactive state while the other is permanently active.

Atrial dissociation ${ }^{24,25)}$ is a phenomenon in which atrial flutter or fibrillation is present in the left atrium and atrial fibrillation or flutter, sinus rhythm or ectopic atrial rhythm is seen in the right simultaneously. A case in which activation of the right atrium is controlled by an intact sinus node and the left atrium shows atrial fibrillation could be thought to be one of the above examples in which the neural network is composed of 2 totally disconnected parts.

Another theory on the mechanism of the initiation and maintenance of atrial fibrillation is ectopic focus hypothesis. It is suitable as a mechanism of the initiation, but not as that of the maintenance.

As shown in Fig. 1, the phases of the $f$ waves of leads aVR and aVL are 
opposite to that of the $f$ waves of lead aVF. From our results of phase angles the excitation wave appeared to descend at least in the right atrium. Both these observations and results are not incompatible with the presence of an ectopic focus or a generator situated at an upper part of the right atrium, and also the assumption of circus movement. Whether either of the 2 assumptions is valid depends upon the other observations and inferences.

Prinzmetal et $\mathrm{a}^{31}$ made simultaneous recording with electrodes separated over $2.5 \mathrm{~cm}$ and could not observe synchronous activation of these electrode locations in esophageal ECGs in man. Sano ${ }^{6)}$ performed similar experiments with intracellular microelectrodes separated over $1.0 \mathrm{~cm}$ in dogs. They denied a circus movement because they could not find synchronous activation of the parts thus explored. On the contrary, it might be rather reasonable that synchronous activation could not be found in simultaneous leads between 2 sites with such distances under the assumption of a circus movement.

Recently Cranefield ${ }^{26)}$ stated that the sustained activity characteristic of fibrillation is not caused by circus movement but the activity of automatic foci, and stressed the latter rather than the former. From our present investigation, however, the number of automatic foci (called generators in our terms) is one and also the presence of circus movement could be assumed though the importance of generator is equally recognized by us as Cranefield did.

Multiple coherences are near unity in every array and partial coherences are often rather large between 2 adjacent sites. These results correspond to the presence of one generator and indicate that of the daughter waves in addition to the mother circus ring.

In order to investigate the properties of random signals, it does not make sense to watch only the momentary values of the data. For that purpose we must grasp their statistical behaviors as a whole. It is necessary to calculate correlation function and/or power spectrum as a measure to estimate the whole properties of the data. Any random signal can be expressed as a sum of sinusoidal waves with various amplitudes and phases and with different frequencies, or as the integral of them. Therefore, the activities of the sinusoidal waves contained on an average in that random signal at each frequency, namely, power spectra are often used as a measure of statistic of this kind. Consequently, the present study laid stress exclusively on power spectrum.

Power spectrum and correlation function compose Fourier transform pair. Formerly, correlation function was first calculated and then power spectrum was derived by transforming it. However, sampling errors in obtaining correlation function often leads to undesirable results. Because of this frequently 
misleading character of the previous method, the present investigation is based upon power spectrum dircctly calculated from the original random data, not through correlation function followed by transformation for spectrum but by means of high speed Fourier transform of the original data, so that correlation function was not studied.

Therefore, direct comparison with the result by Battersby'11) who calculated correlation function of the $f$ waves and found the periodicity of these waves could not be made. However, it may be considered that similar results were obtained as to the presence of a pacemaker (generator) and its rate.

\section{REFERENGES}

1. Moe GK: A conceptual model of atrial fibrillation. J Electrocardiol 1: 145, 1968

2. Abildskov JA, Millar K, Burgess MJ: Atrial fibrillation. Am J Cardiol 28: 263, 1971

3. Prinzmetal N, Corday E, Brill IC, Oblath RW, Kruger HE: The Auricular Arrhythmias. Charles C Thornas, Springfield, I1l, 1952

4. Sano T, Scher AM: Multiple recording during electrically induced atrial fibrillation. Circulat Res 14: 117, 1964

5. Scherf D, Cohen J: The Atrioventricular Node and Selected Cardiac Arrhythmias. Grune \& Stratton, New York, 1964

6. Sano T: Mechanism of fibrillation. in Symposium on Cardiac Arrhythmias, ed by Sand $\phi \mathrm{e}$ E, Flensted-Jensen E, Olesen KH, Elsinore, AB Astra, p 25, 1970

7. Scherf D, Schott A: Extrasystoles and Allied Arrhythmias. 2nd Edition, William Heinemann, London, 1973

8. Walter DO, Adey WR: Analysis of brain-wave generators as multiple statistical time series. IEEE Trans Biomed Engineering BME 12: 8, 1965

9. Blackman RB, Tukey JW: The Measurement of Power Spectra. Dover, New York, 1958

10. Rytand DA: The circus movement (entrapped circuit wave) hypothesis and atrial flutter. Ann Int Med 65: 125, 1966

11. Lewis T, Feil HS, Stroud WD: Observations upon flutter and fibrillation. Part II. The nature of auricular flutter. Heart 7: 191, 1920

12. Lewis T: The Mechanism and Graphic Registration of the Heart Beat. 3rd Edition, Shadow \& Sons, London, p341, 1925

13. Lewis $\mathrm{T}$, Drury AN, Iliescu GC: A demonstration of circus movement in clinical flutter of the auricles. Heart 8: 341,1921

14. Sano $T$, Suzuki F, Sato S: Sinus node impulses and atrial fibrillation. Circulat Res 21 : 507,1967

15. Nadeau RA, Roberge FA, Billette J: Role of the sinus node in the mechanism of cholinergic atrial fibrillation. Circulat Res 27: 129, 1970

16. Phair WB: Familial atrial fibrillation. Canad Med Ass J 89: 1274, 1963

17. Penfield W, Jasper H: Epilepsy and the Functional Anatomy of the Human Brain. J \& A Churchill, London, p470, 1954

18. Wiener N, Rosenblueth A: The mathematical formulation of the problem of conduction of impulses in a network of connected excitable elements, specifically in cardiac muscle. Arch Inst Cardiol México 16: 205, 1946

19. Farley BG, Clark WA: Activity in networks of neuron-like elements. in Information Theory, 4th London Symposium, ed by Cherry C, Butterworths, London, p242, 1961 
20. Farley BG: Some results of computer simulation of neuron-like nets. Fed Proc 21: 92 , 1962

21. Farley BG: Some similarities between the behaviour of a neural network model and electrophysiological experiments. in Self-organizing Systems, ed by Yovits MC, Spartan Books, Washington, p535, 1962

22. Moe GK, Rheinboldt WC, Abildskov JA: A computer model of atrial fibrillation. Am Heart J 67: 200, 1964

23. Griffith JS: Mathematical Neurobiology. An Introduction to the Mathematics of the Nervous System. Academic Press, London, p 67, 1971

24. Bellet S: Clinical Disorders of the Heart Beat. Lea \& Febiger, Philadelphia, 1971.

25. Chung EK: Principles of Cardiac Arrhythmias. Williams \& Wilkins, Baltimore, 1971

26. Cranefield PF: The Conduction of the Cardiac Impulse. Futura, Mount Kisco, New York, 1975

27. Battersby EJ: Pacemaker periodicity in atrial fibrillation. Circulat Res 17: 296, 1965 